\title{
Topology Optimisation: Increasing the Speed and Reliability of Design
}

\author{
Liam Kelly* $^{*}$ Andy Keane ${ }^{\dagger}$, András Sóbester* and David Toal ${ }^{\S}$ \\ University of Southampton, UK, SO16 7QF
}

\begin{abstract}
In this paper, topology optimisation is applied to the design of the rear fuselage of an unmanned aerial vehicle (UAV). A comparison is drawn between the performance of a design created through evolutionary structural optimisation (ESO) and a baseline design modelled on a manually designed and successfully flow fuselage geometry, for different wing shapes. The loading for each wing shape is determined by full-potential (FP) aerodynamic analysis. A Kriging model is then employed in a multidisciplinary optimisation procedure driving a trade study between aerodynamic efficiency and aircraft structural weight. Using this procedure, a Pareto front is populated to give a set of optimal designs which satisfy maximum aerodynamic efficiency and minimum weight objectives. A wide search of the design space is achieved with little manual intervention, which makes use of the high fidelity weight estimate extracted from topology optimization results.
\end{abstract}

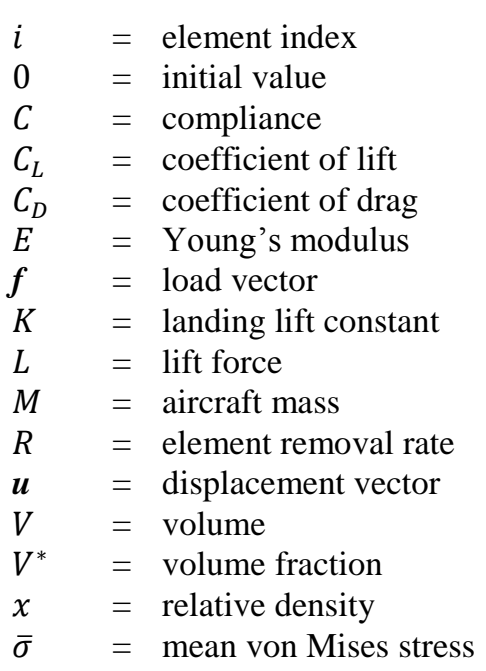

\section{Nomenclature}

\section{Introduction}

JCREASINGLY, complex design systems are operated from a range of different computational platforms with a number of different design teams. The more complex these systems become, the less likely it is to see effective integration of each of these specialist activities ${ }^{1}$. This often results in the opportunities to change the design decreasing dramatically the further one progresses through the design process, and often this allows imperfections to remain in the design as it is too expensive to go back and correct them ${ }^{2}$. Better integration within the design process can facilitate smaller design organisations with shorter time to manufacture and less human interaction. By more fully exploiting the capabilities of computers in design, search and optimisation, this is an achievable objective. The increasing power of modern computer systems continues to expand the depth of computational analysis that can be afforded within the design process.

\footnotetext{
*PhD Candidate, Rolls-Royce UTC for Computational Engineering, liam.kelly@ soton.ac.uk.

${ }^{\dagger}$ Professor of Computational Engineering, Aeronautics, Astronautics and Computational Engineering.

* Senior Lecturer, Aeronautics, Astronautics and Computational Engineering.

$\S$ New Frontiers Fellow, Aeronautics, Astronautics and Computational Engineering.
} 
As part of the DECODE ${ }^{* *}$ project, Quaranta et al. presented a method whereby aerodynamic optimisation is integrated into the concept design stage of an Unmanned Aerial Vehicle (UAV) ${ }^{3}$. The aerodynamic optimisation is carried out using an estimate for the weight of the structure based on previous models. Commonly, statistical models are used to provide weight estimates to inform optimisation decisions, some of which are reviewed in Ref. 4. To manually update the internal structure in this model would take many hours of Computer Aided Design (CAD) drawing, carried out by a skilled design engineer. Automation of the internal design definition would permit a high fidelity weight estimation, allowing the designer to conduct a much more accurate search of the design space with little additional manual effort.

In the literature, several authors have used higher fidelity geometry-generation methods capable of providing weight estimates and structural analysis for the multidisciplinary optimisation of aircrafts. In Ref. 5, an overengineered structural universe of stiffening ribs is automatically generated for UAVs from which the final stiffening pattern can be selected. Stiffener configurations are manually selected from this universe, and structural analysis is used to ensure that the configuration is sufficient for a given wing geometry. The most common method for automated generation and sizing of internal geometry is by parametric definition - allowing the creation, resizing or re-location of stiffening features within the design. One such example is the OptWing code, which allows rapid model generation and mapping to analysis models based on a parametric description ${ }^{6}$.

Here, evolutionary topology optimisation gives rise to another automated approach to generating an internal structure automatically from a specified design domain. Topology optimisation allows a much wider search of the design domain since it is free from the limitations of a parametric definition. As such, it has been implemented in the redesign of many aerospace parts to decrease mass ${ }^{7-9}$. Ref. 10 takes a similar approach to that applied here: there a two-level optimisation approach is applied to an engine pylon. At the top level, the external geometry is optimised, while at the bottom level, topology optimisation is used to generate an optimal structure for given displacement constraints.

In the past, the ability to manufacture the freeform designs generated by topology optimisation has provided an obstacle in the path of implementing optimisation results. Now, due to the advances in additive manufacturing (AM) technology, these complex or feature rich geometries can be readily manufactured ${ }^{11}$. AM is now capable of creating end-use production components, as opposed to just one-off prototypes ${ }^{\dagger \dagger}$. The higher cost of AM versus conventional manufacturing techniques can be offset by the increased performance of complex parts, making it most applicable to high performance applications such as aerospace, automotive and medical applications.

In this paper, a UAV example is used to demonstrate the power of topology optimisation, specifically the Bidirectional Evolutionary Optimisation (BESO) algorithm ${ }^{12}$, in design search. A trade study is carried out between structurally optimised concept designs to determine which design gives the greatest aerodynamic efficiency (liftto-drag ratio) for the lightest aircraft. The aim is to use topology optimisation to automatically generate a sufficient structural design to provide a high-fidelity weight and performance estimate to inform multi-disciplinary optimisation.

\section{Topology Optimisation}

The topology optimisation algorithm used here is a BESO algorithm adapted from Ref. 12. A basic overview of the BESO method is shown Figure 1. Commonly, the evolutionary structural optimisation algorithm is used for optimisation problems which aim to determine a minimum compliance (maximum stiffness) structure for a given volume constraint ${ }^{13}$. In this paper, an alternative method is also used to drive the optimisation to a minimum

Table 1 - Overview of optimisation problems

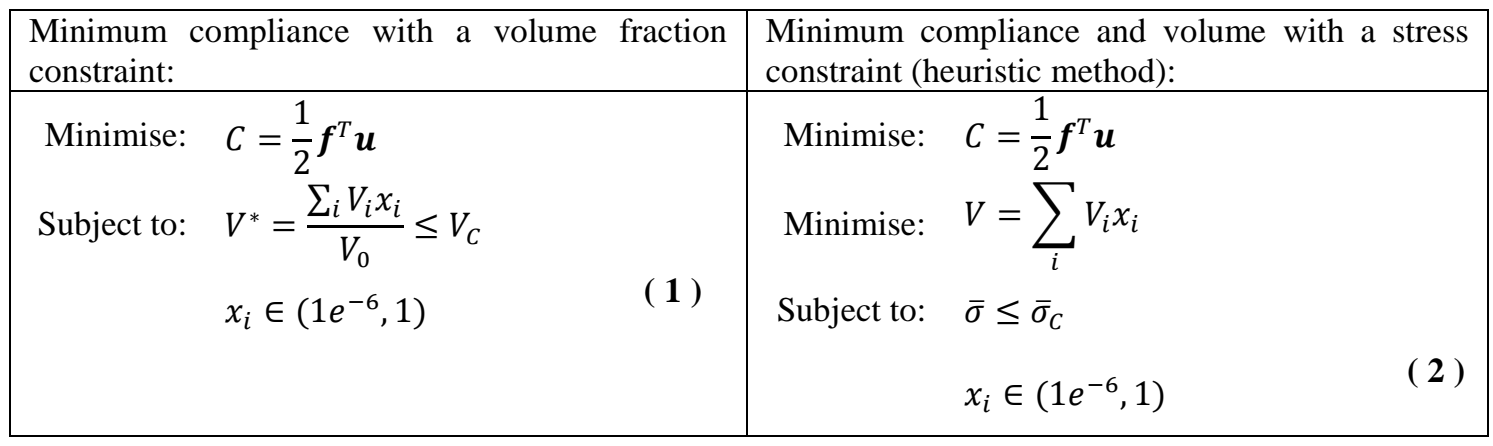

\footnotetext{
** Decision Environment for Complex Engineering Design -

[Online: http://www.southampton.ac.uk/ decode/ (Accessed: 24 October 2013)]

† 3T RPD - [Online: http://www.3trpd.co.uk/ (Accessed: 15 April 2014)]
} 
volume, minimum compliance design with a mean von Mises stress constraint. Table 1 describes the general optimisation problem for each example.

There are a number of topology optimisation methods which could have been used here: boundary methods, such as level set optimisation, or density-based methods, such as SIMP (Solid Isotropic Material with Penalization) optimisation, are just two examples. These methods are reviewed extensively in Ref. 14. The BESO algorithm was chosen due to the ease with which it can be implemented alongside commercial software and adapted to suit the needs of the project. The elemental Young's modulus is then scaled using Eq. ( 3 ).

$$
E_{i}=E_{0} x_{i}
$$

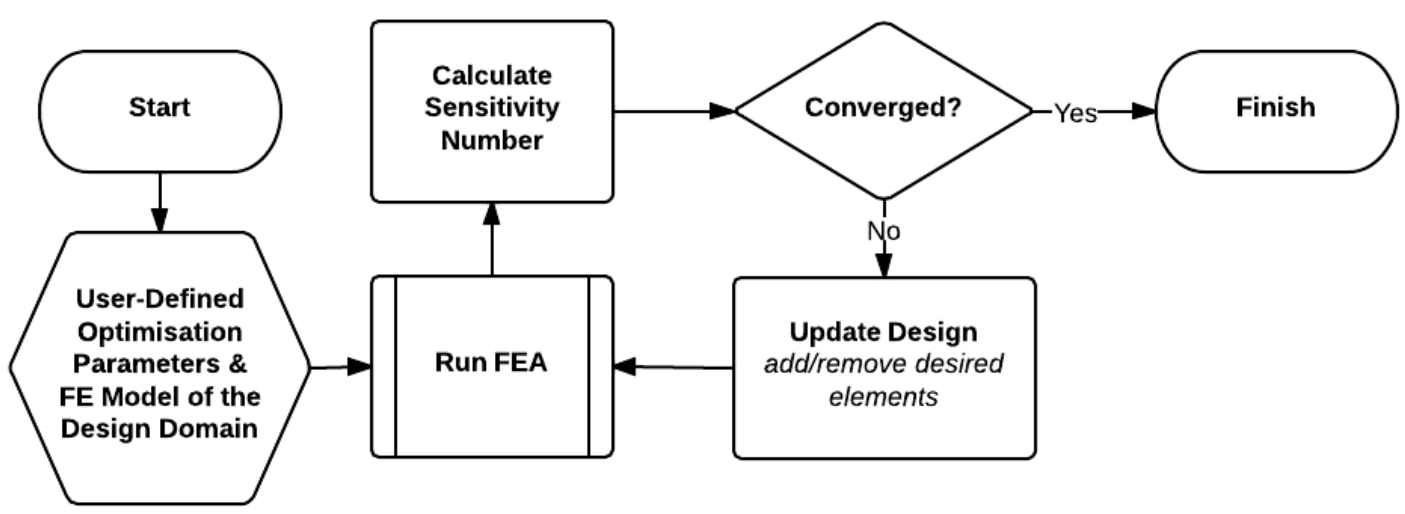

Figure 1 - BESO algorithm

The BESO algorithm used is set up as in Ref. 15. In this paper, the algorithm is used to find a minimum compliance structure with a volume constraint, as described below. Here, two adaptations have been made:

- removal of the history averaging equation (eq. 6 in Ref. 15) - averaging the sensitivity number over previous iterations may help to stabilise the evolutionary process but can also give the optimiser a distorted view of the current state of the structure,

- adaptation of the filter scheme (eq. 3 in Ref. 15) to average a fixed number of neighbouring elements as opposed to a fixed radius, this is done to increase computational efficiency, with only small effect on the result.

When implementing a mean von Mises stress constraint, the target volume fraction at each iteration is determined by comparison of the current mean von Mises stress with the von Mises stress limit, as in Eq. ( 4 ).

$$
V^{*}=\left(\frac{\sum_{i} V_{i} x_{i}}{\sum_{i} V_{i}}\right) \times\left\{\begin{array}{lll}
\max \left[\frac{\bar{\sigma}_{C}-\bar{\sigma}}{\bar{\sigma}_{C}}, 1-R\right] & \text { when } & \bar{\sigma}_{C} \geq \bar{\sigma} \\
\min \left[\frac{\bar{\sigma}_{C}-\bar{\sigma}}{\bar{\sigma}_{C}}, 1+R\right] & \text { when } & \bar{\sigma}_{C}<\bar{\sigma}
\end{array}\right.
$$

The element removal rate $(R)$ determines the maximum volume fraction to be removed in each iteration. The design update decisions are made based on the strain energy density of each element, as described in Ref. 15. The reason for using the mean von Mises stress as opposed to the maximum is because it is much more stable over the course of the optimisation. The maximum stress can be greatly affected by any change in topology and can jump between different locations, making it difficult to achieve convergence.

\section{Design Architecture}

A typical design cycle comprises three main stages: concept, preliminary, and detailed design. In the concept design stage, a number of competing designs are often generated to provide an insight into whether it is feasible to continue with the design process. In most design organisations, the selection of the best concept to take forwards relies on experienced human comparison, without fully exploiting the capabilities of modern search and optimisation strategies ${ }^{2}$.

In the preliminary stage, this concept design is commonly broken up amongst a number of dedicated teams. For example, a team may be assigned to each component (e.g. wings, undercarriage etc.) or perhaps each discipline (e.g. aerodynamics, structures etc.). It is at this stage that topology optimisation can have the most impact on structural design. The presence of powerful High Performance Computing (HPC) clusters gives the opportunity to run a number of individual structural topology optimisations in parallel to automatically carry out structural 
preliminary design for a number of concepts. Note that a number of other decisions must be made at this stage for other aspects of the design (e.g. component choices), though these decisions may be influenced by the output of structural design.

Following selection of the best structural design, final CAD details can be created to prepare the part for manufacture. A schematic of the suggested structural design cycle is shown in Figure 2. Difficulty arises in this approach when deciding what level of detail is required in the model put forward for topology optimisation; i.e., which features should be included and which can be considered to have negligible impact on the structural design and therefore added in at a later stage?

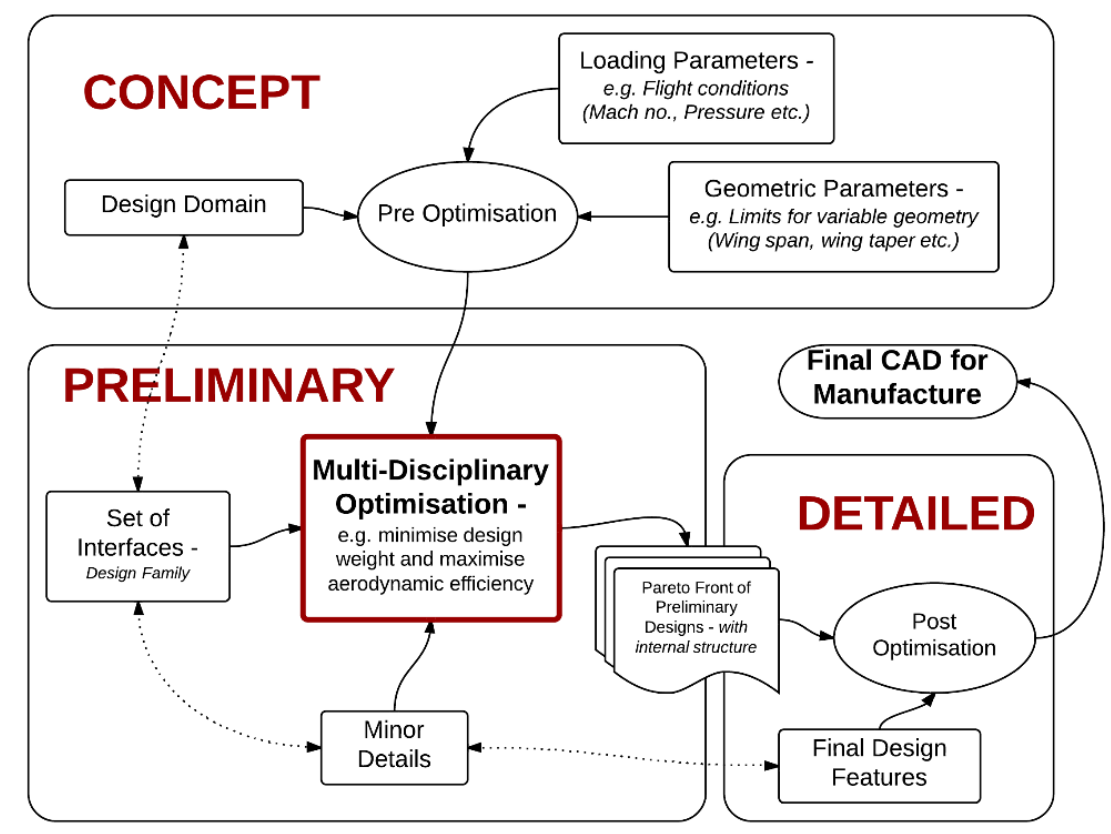

Figure 2 - Design cycle including a topology optimisation trade study

\section{UAV Application}

To demonstrate the use of topology optimisation within a design trade study, an unmanned aerial vehicle (UAV) application has been used. At the University of Southampton, a number of lightweight laser-sintered UAVs have been designed as part of the DECODE project, allowing a range of comparisons to be drawn between topology optimised structures and those with a manually designed internal structure.

The use of multi-disciplinary optimisation methods in the preliminary design of aircraft and UAVs is becoming increasingly commonplace. In aircraft design, there are a number of disciplines which need to collaborate effectively in order to create a successful design.

Figure 4 gives a suggestion of possible interactions between structures, aerodynamics and propulsion disciplines as suggested in Ref. 2. Typically in early stage design, trades are made using low fidelity analyses and weight estimates from

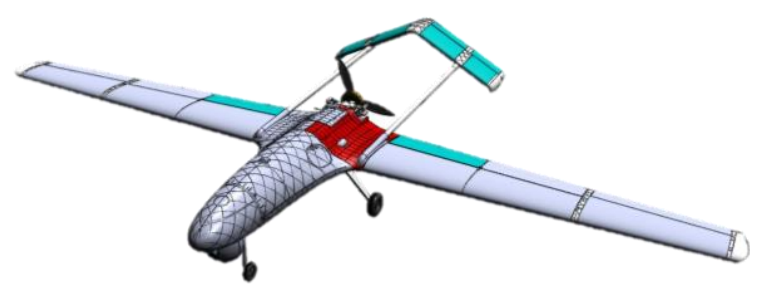

Figure 3 - CAD drawing of DECODE Mark V statistical models and empirical relationships or perhaps parametric models. By introducing topology optimisation into the early stage design trade studies, the aim is to increase the fidelity of multi-disciplinary optimisation at this stage and generate a more optimal set of solutions by allowing a broader and more accurate search of the design space.

The part considered for optimisation here is the rear section of a fuselage taken from a DECODE aircraft (shown in red in Figure 3). This aircraft has a maximum take-off weight (MTOW) of $25 \mathrm{~kg}$, and has an additive manufactured nylon fuselage structure and ultra-light foam core aerodynamic surfaces. The aircraft is designed to carry a $5 \mathrm{~kg}$ payload, with an 8 hour endurance. The manually designed fuselage is a monocoque structure, 


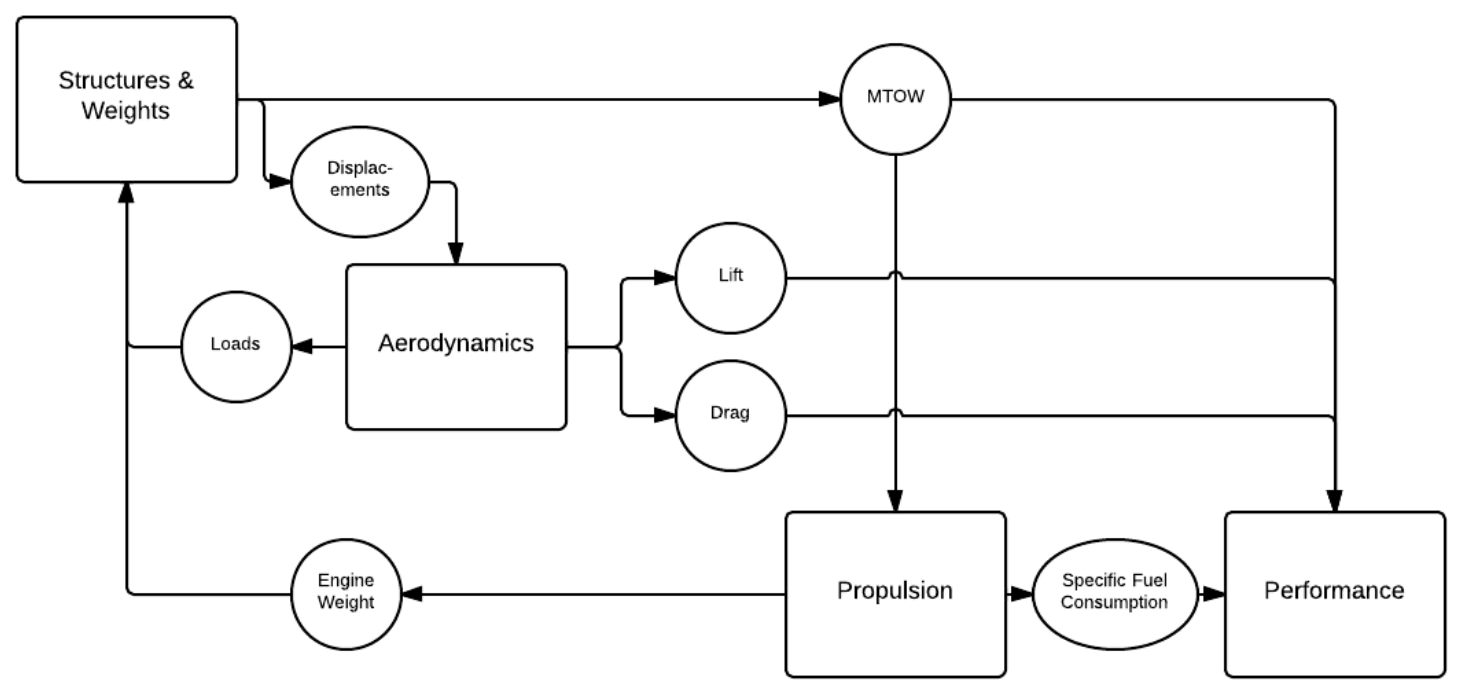

\section{Figure 4 - A suggestion of multi-disciplinary interactions}

stiffened by a series of internal ribs. The aims in this project are two-fold: firstly, to generate a more efficient structural design which will fully exploit the design freedom of additive manufacture, and secondly to use this automated design process to provide high fidelity structural weight estimates for preliminary design trade studies paying particular attention to structural weight versus performance trades.

\section{A. Design Domain}

In setting up the design domain for optimisation, the design features of the fuselage are classified here into three categories: design interfaces, minor details and final design features. These classifications and a description of each group can be seen in Table 2. Taking these classifications into account, the assembly for optimisation is shown in Figure 5. In order to decrease the computational expense, the domain is assumed to be symmetrical so that only half of the domain requires analysis.

\section{B. Loading}

Due to the constantly evolving design domain, the application of loads and boundary conditions must be thoughtfully applied to ensure that they will remain appropriate throughout the course of the topology

\section{Table 2 - Classification of design features}

\begin{tabular}{|l|l|l|}
\hline Classification & Features & Description \\
\hline Interfaces & $\begin{array}{l}\text { Spars } \\
\text { Wings } \\
\text { Engine } \\
\text { Undercarriage } \\
\text { Front }\end{array}$ & $\begin{array}{l}\text { Interfaces are features which have an effect of the } \\
\text { loading of the structure and in turn are likely to } \\
\text { have an impact on the structural design. }\end{array}$ \\
\hline Details & $\begin{array}{l}\text { Fuel Tank } \\
\text { Power Unit } \\
\text { Engine } \\
\text { Undercarriage } \\
\text { Spars }\end{array}$ & $\begin{array}{l}\text { Details refers to parts which require consideration } \\
\text { in the geometry of the design domain. These } \\
\text { features may or may not also be classified as } \\
\text { interfaces. }\end{array}$ \\
\hline Final Features & $\begin{array}{l}\text { Cowling } \\
\text { Power unit vents } \\
\text { Engine vents } \\
\text { Fuselage interface } \\
\text { Wing interface } \\
\text { Servos \& wiring } \\
\text { Tail spar mount } \\
\text { Component interfaces }\end{array}$ & $\begin{array}{l}\text { Final features are the final attachments and } \\
\text { connections which will have negligible effect on } \\
\text { the structural macrostructure of the part and are } \\
\text { often impossible to place before the structure of } \\
\text { the part is designed. }\end{array}$ \\
\hline
\end{tabular}


optimisation. This is often carried out by removing loaded and constrained surfaces from the changeable design domain in order to ensure that they are not deleted from the structural analysis during the optimisation.

Since the structure to be optimisation here is part of an assembly, the interactions between parts must also be carefully considered. As selected above, the part will interact with the spars and wings, and with the engine, undercarriage and front fuselage. In this instance, a tie constraint has been used to connect the fuselage to the wings and spars in the interest of computational speed. The engine, undercarriage and front fuselage are each modelled as point masses which are coupled with the surface of the fuselage in designated areas. Since maintaining bolt holes throughout the optimisation process can be problematic, the bolt holes are modelled as square regions on the surface of the domain

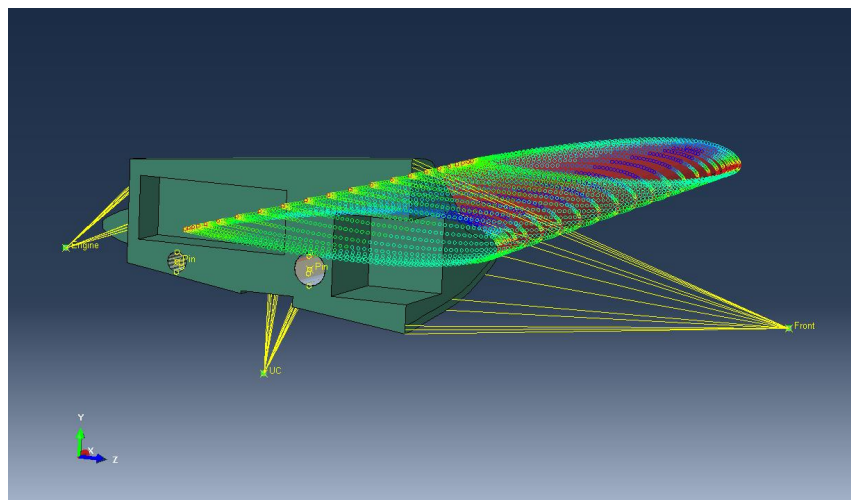

Figure 5 - Fuselage and wing assembly (symmetric halfdomain) (as can be seen in Figure 5).

Wing loading is determined by directly mapping a pressure profile generated using a full-potential (FP) analysis ${ }^{16}$ with viscous drag correction ${ }^{17}$. The FP method determines the coefficient of pressure across the surface of the wing for a given wing size and Mach number. The angle of attack of the wing is adjusted to provide the required lift. In this example, only loading at a cruise speed and altitude has been considered. In practice, it may be advisable to consider other load cases such as landing, manoeuvre, transportation loads.

Table 3 includes the magnitude of the loads used in this example. This includes the approximate mass of all components taken from the as-flown DECODE aircraft. The magnitudes given are $50 \%$ of the total weight as they are reflected in the symmetry plane.
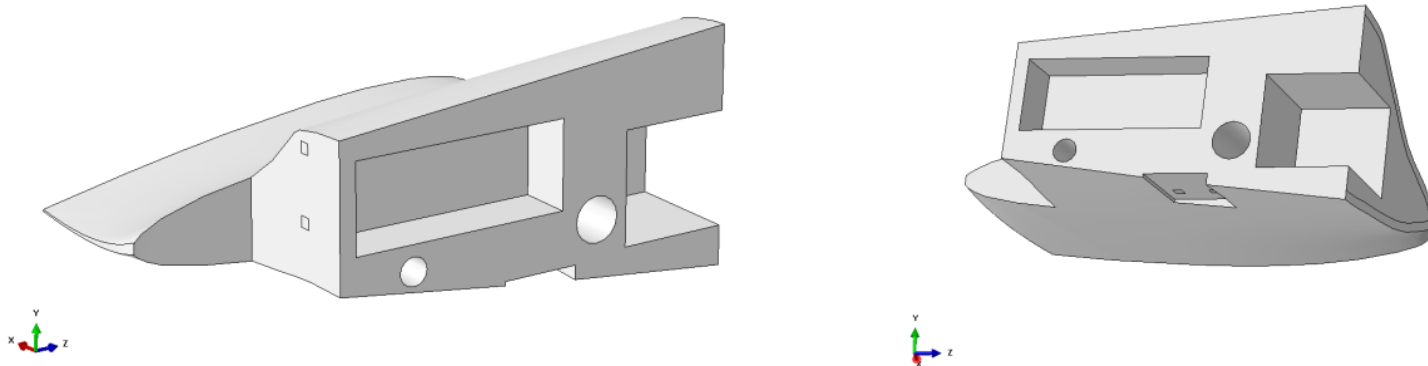

Figure 6 - Fuselage part for optimisation

\section{Topology Optimisation}

In this section, topology optimisation is carried out on the design domain, described above, and compared to a baseline model (Figure 7). The baseline model is adapted from the fuselage which was successfully manufactured and flown as part of the DECODE project. The part has been simplified to remove any final design details and to make the part symmetrical. The volume of the baseline model fills approximately $6 \%$ of the design domain, therefore a minimum compliance optimisation is carried out with a volume fraction constraint of $6 \%$.

The wings are parameterised using three variables, as shown in Figure 8: taper, twist and semi-span normalised by the root chord. Here, the sweep angle will be fixed at $2^{\circ}$. The baseline and optimised part will be compared for 10 different wing geometries, generated by a space-filling design of experiments (DOE), the boundaries for which are shown in Table 4. 
Table 3 - Magnitude and position of rear fuselage loading

\begin{tabular}{|l|l|l|}
\hline Load & Magnitude (N) & Location (origin at spar) \\
\hline Wing Loading & $\begin{array}{l}\text { Determined by wing pressure } \\
\text { mapped from FP }\end{array}$ & Wing surface \\
\hline Wing \& Spar weight & Dependant on wing geometry & Self-weight of elements \\
\hline Rear Fuselage weight & $V^{*} \times 9.822 \mathrm{~g}$ & Self-weight of elements \\
\hline Front Section of Aircraft weight & $5 \mathrm{~g}$ & \\
\hline Engine weight & $1.75 \mathrm{~g}$ & \\
\hline Undercarriage weight & $0.5 \mathrm{~g}$ & Added to mass not load \\
\hline Fuel weight & $0.6 \mathrm{~g}$ & Added to mass not load \\
\hline Power Unit weight & $0.75 \mathrm{~g}$ & Added to mass not load \\
\hline Tail weight & $0.75 \mathrm{~g}$ &
\end{tabular}

The driving constraint aerodynamically is that the aircraft must be able to fly at landing speed. This constraint is monitored using the ratio between the maximum lift achievable (assumed to be at an angle of attack of $15^{\circ}$ ) at landing speed and the required lift (aircraft weight), as shown in Eq. ( 5 ).

$$
\frac{L_{\text {landing_max }}}{L_{\text {required }}} \geq K
$$
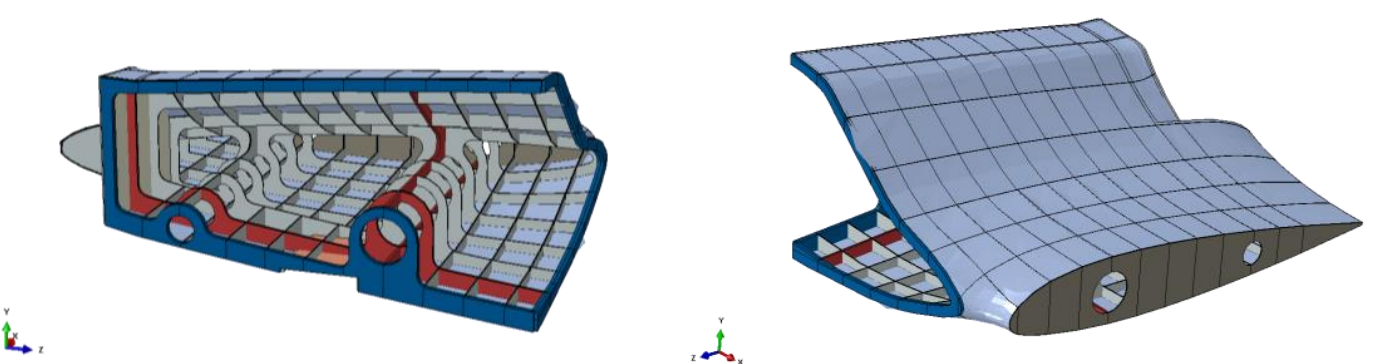

Figure 7 - Baseline Shell Model
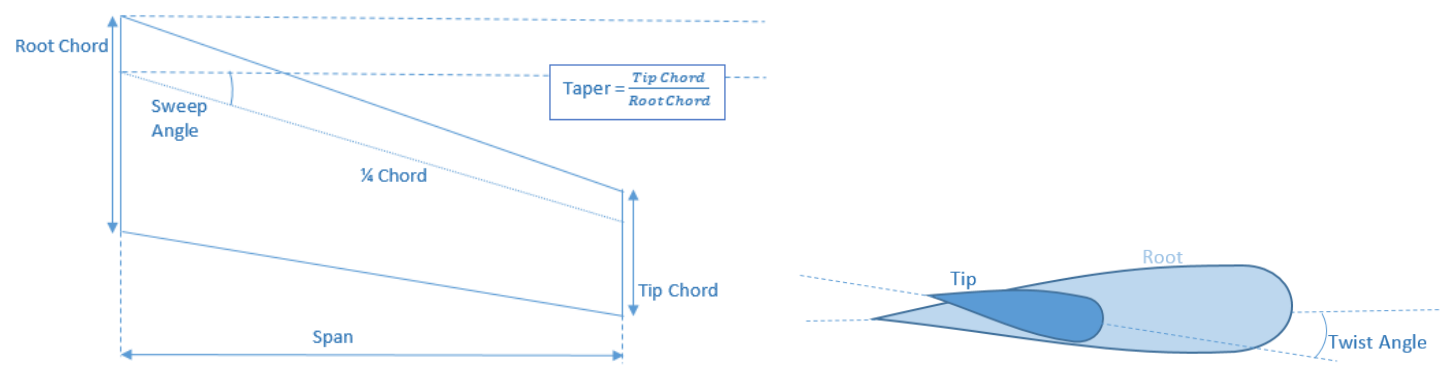

Figure 8 - Wing Geometry Parameters

Table 4 - Preliminary Variable Limits

\begin{tabular}{|l|l|l|}
\hline Variable & Lower & Upper \\
\hline Normalised semi-span & 2 & 4 \\
\hline Twist & $-2^{\circ}$ & $2^{\circ}$ \\
\hline Taper & 0.7 & 1 \\
\hline Sweep (fixed) & 2 & 2 \\
\hline
\end{tabular}

Analysis of the results in Figure 10 show that for each wing geometry, the compliance of the optimised fuselage is less than that of the baseline fuselage. The average reduction in compliance is approximately $52.7 \%$. 


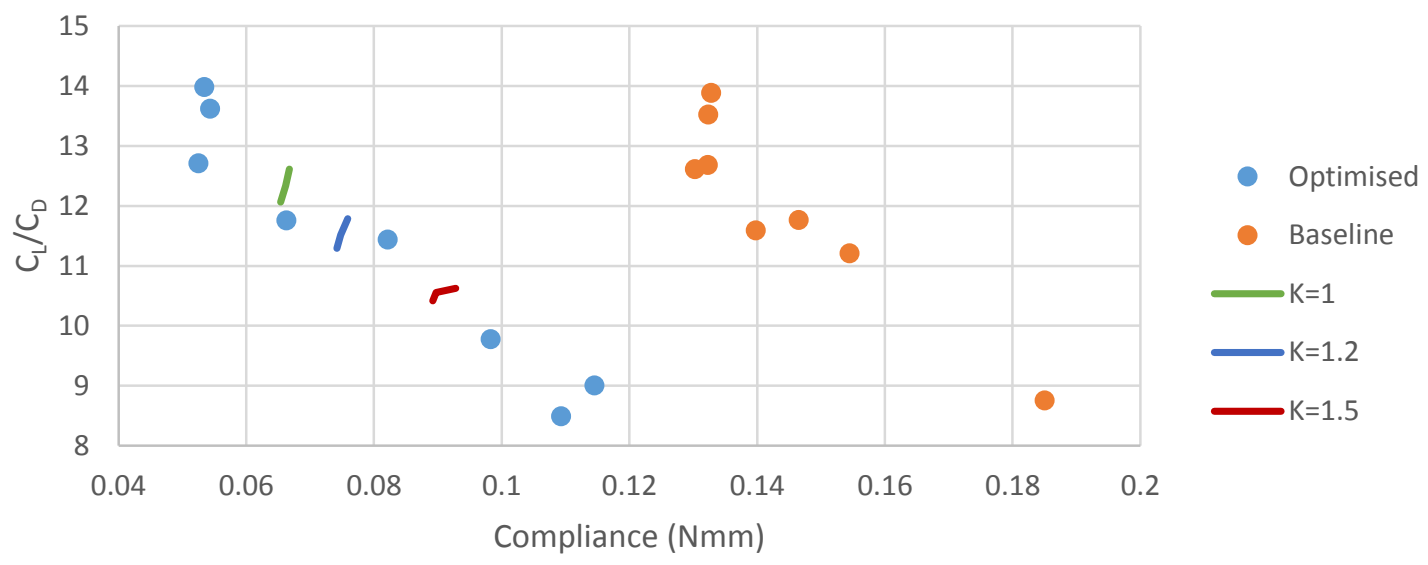

Figure 10 - Compliance vs. CL/CD for Optimised Fuselage and Baseline Fuselage
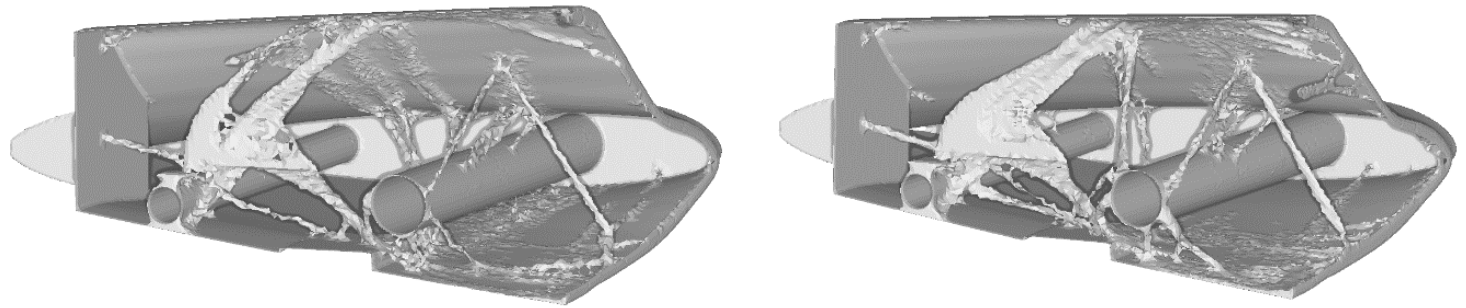

Figure 9 - Topology optimisation results for two different wing geometries

Also included on the figure is an estimate of the constraint boundary, at a $K$ value of $1,1.2$ and 1.5. These estimated curves are generated by interpolating the constraint value for the available data from the 10 geometries tested. The final geometry of two different fuselage sections can be seen in Figure 9. Though both parts are the same weight, the topology is different to suit the variation in loading between the two different wing geometries. A stress plot of the baseline and optimised model can be seen for one case in Figure 11. It is notable that the maximum stresses in the baseline model are much higher (approximately 10x) and that in the baseline model a much higher stress can be seen in the skin of the structure, even though the skin shell thickness is the same in each example. Topology optimisation has therefore directed the design of the part away from a monocoque structure towards a more space frame structure where the load is carried by supporting struts rather than through the skin.

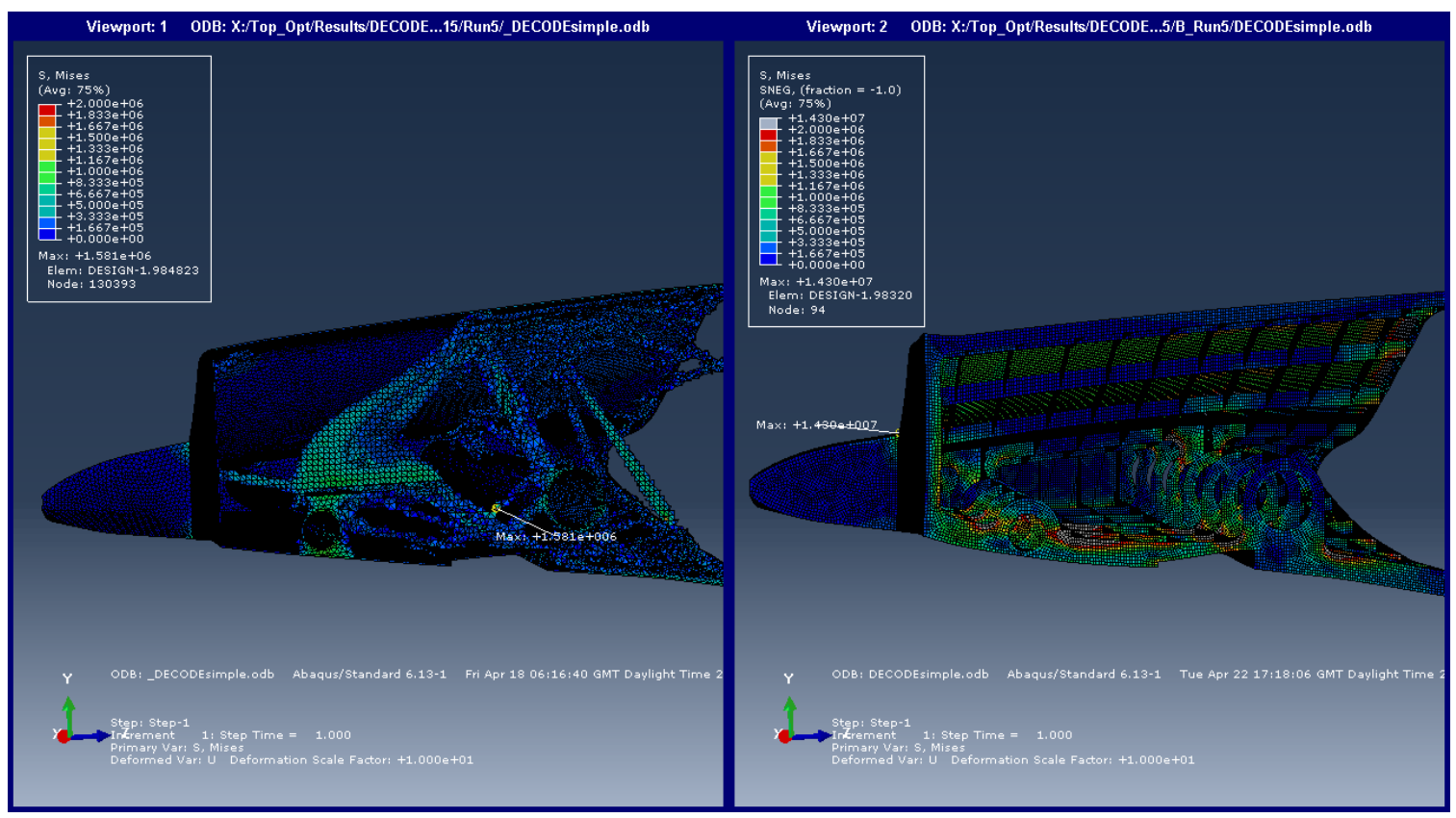

Figure 11 - von Mises stress plot for the optimised model (left) and the baseline shell model (right) 


\section{Trade Study}

Based on the preliminary tests above, a mean von Mises stress limit of 0.5MPa has been assumed. This choice has been made arbitrarily based on the mean stress in each of the designs generated in the previous section. The upper and lower bounds for the wing geometry parameters are set as in Table 4. The multi-disciplinary optimisation problem to be solved by this trade study is shown in Eq. ( 6 ).

$$
\begin{aligned}
\text { maximise: } & \frac{C_{L}}{C_{D}} \\
\text { minimise: } & M \\
& \frac{L_{\text {landing_max }}}{L_{\text {required }}} \geq 1.2 \\
\text { subject to: } & \bar{\sigma} \quad \leq 0.5 \times 10^{6}
\end{aligned}
$$

The aircraft mass $(M)$ is calculated by adding the optimised rear fuselage mass to a fixed estimate for the rest of the empty weight of the aircraft and an estimate for the wing and spars which varies by volume, based on a fixed material density for the carbon spar and foam wing.

To begin each function evaluation, a fuselage mass is assumed at $7.5 \%$ of the permissible fuselage design domain in order to estimate the wing loading from FP analysis. Upon convergence of topology optimisation, the mass estimate is updated and FP analysis is repeated before continuing the topology optimisation. This process continues until convergence. An overview is shown in Figure 12.

The initial search of the design space is carried out using a 10 point space-fill DOE. A response surface is then built using a kriging model, and improvements are sought using a mixture of updates strategies based on the optimum, expected improvement and root mean squared error. This design search is made using OPTIMAT v2 for Matlab $\$$.

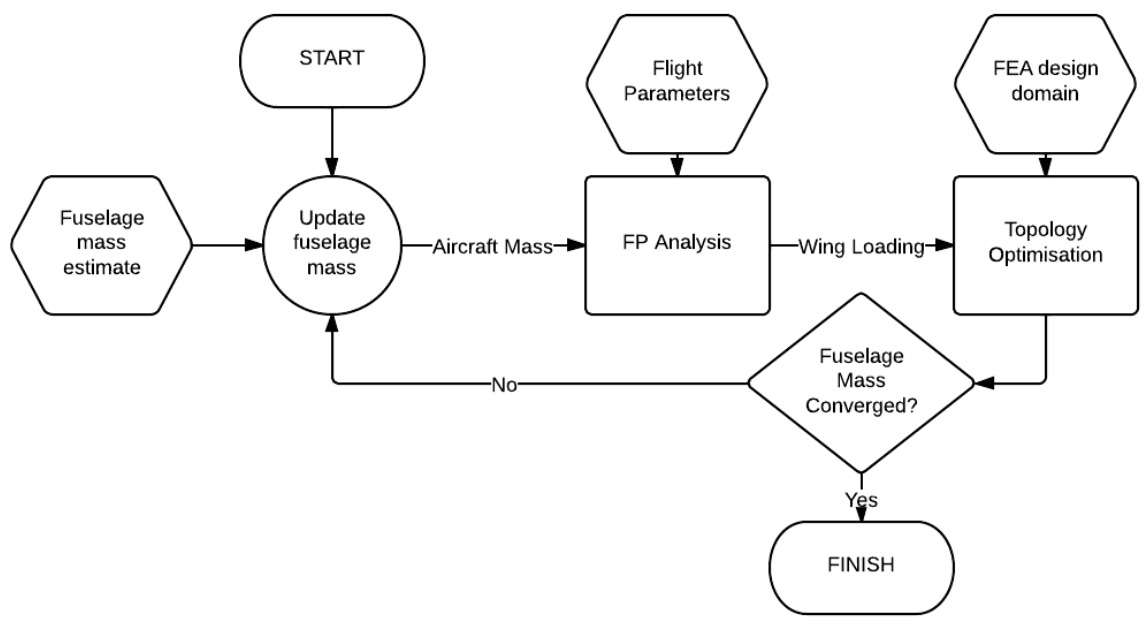

\section{Figure 12 - Multidisciplinary optimisation procedure}

Figure 13 shows the results of topology optimisation runs for 50 sets of design parameters. The total aircraft weight varies between $15.88 \mathrm{~kg}$ and $16.89 \mathrm{~kg}$ dependant on the size and shape of the wing. The rear fuselage mass varies between $56.4 \mathrm{~g}$ and $83.3 \mathrm{~g}$. The main area of interest is magnified in Figure 14. The highlighted points show four suggested optimal geometries with minimum weight and maximum aerodynamic efficiency, with the orange line highlighting the Pareto front. The infeasible points do not meet the prescribed constraint on lift at landing speed. It is of note that the position of the Pareto front will vary dependant on the safety factor included on the lift constraint. A safer design will be heavier and less aerodynamically efficient, and vice versa. It can also be seen from Figure 13 that a reasonably strong linear correlation can be seen between aerodynamic efficiency and mass. This may be due to the fixed root chord of the wing resulting in an almost linear relationship between span and wing area (and therefore mass). Similarly, the use of linear elastic analysis may cause the fuselage mass to increase linearly with load.

The design parameters for the four optimal points are shown in Table 5. The results suggest that a tapered wing is beneficial to minimise the wing mass, while maintaining aerodynamic efficiency. The results also suggest

\# OPTIMAT v2 is a Rolls-Royce multidisciplinary optimization package developed at the University of Southampton. 
that a normalised semi-span of between 2.892 and 2.985 is most optimal. The mass of the fuselage section for these points varies between $65.1 \mathrm{~g}$ and $65.7 \mathrm{~g}$.

Table 5 - Pareto-optimal Wing Parameters

\begin{tabular}{|l|l|l|l|l|l|l|l|}
\hline Span & Taper & Twist & Sweep & Fuselage Mass $(g)$ & Total Mass $(\mathbf{k g})$ & $\mathbf{C}_{\mathrm{L}} / \mathbf{C}_{\mathbf{D}}$ & Constraint \\
\hline 2.985 & 0.7 & -0.2 & 2 & 65.6 & 16.23 & 12.1527 & 1.2001 \\
\hline 2.894 & 0.7 & 2.0 & 2 & 65.7 & 16.21 & 12.1046 & 1.2003 \\
\hline 2.892 & 0.7 & 2.0 & 2 & 65.7 & 16.22 & 12.1088 & 1.2005 \\
\hline 2.977 & 0.7 & 0.0 & 2 & 65.1 & 16.22 & 12.1249 & 1.2006 \\
\hline
\end{tabular}

Currently, at this level of detail, each topology optimisation run takes of the order of 18 hours to run on a 16 core machine with 64GB RAM - using the high performance computing cluster at the University of Southampton, a number of these optimisation runs can be carried out in parallel. So with an initial DOE and a typical three/four sets of updates, the total bi-level multi-disciplinary design optimisation takes around 4 days. A broad search of the design space is carried out in this time, and very little manual intervention is required.

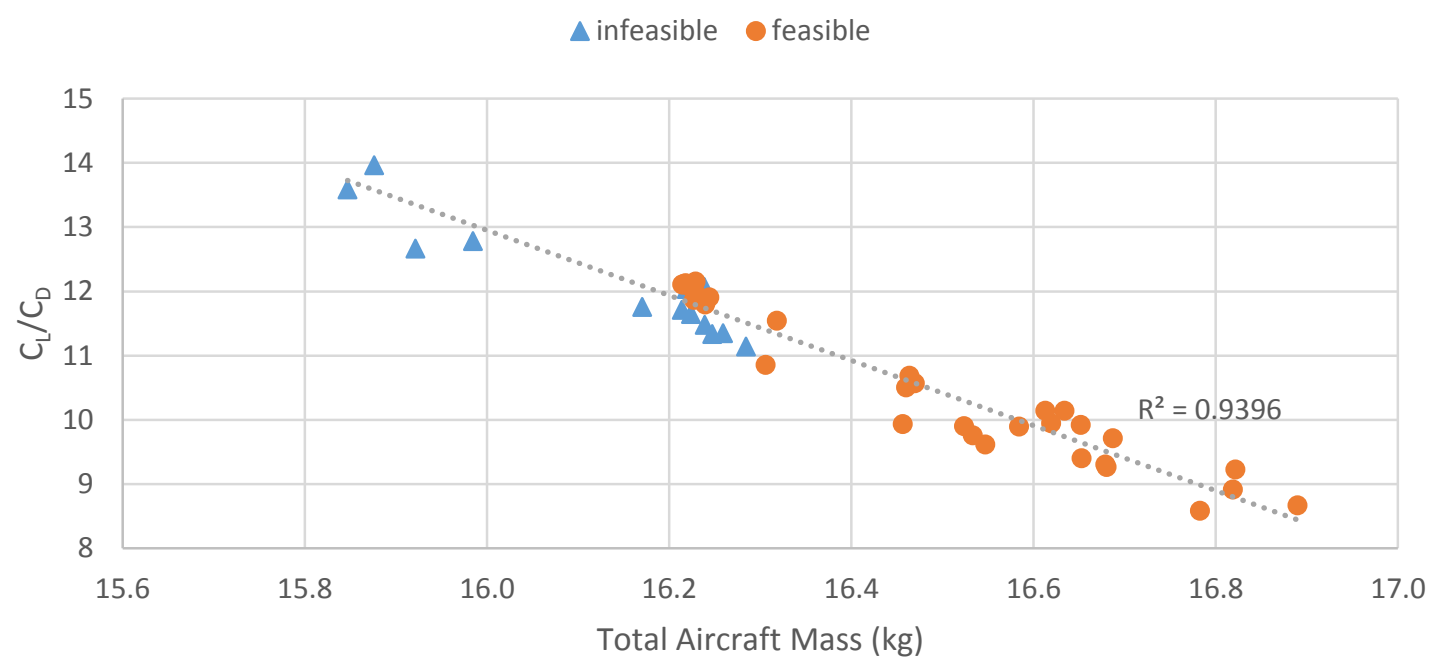

Figure 13 - Total Aircraft Mass vs. Aerodynamic Efficiency (50 points)

\section{Conclusion \& Future Work}

In the present paper, topology optimisation has been used to examine early design stage estimates for the internal structure and design weight of a section of a UAV fuselage. These estimates inform a trade study comparing the aerodynamic efficiency $\left(C_{L} / C_{D}\right)$ to the structural mass of the aircraft. A kriging model is used to discover four points along a Pareto front, from which suggestions of the optimal wing geometry can be made. For each of these points, a fuselage internal structure is generated to provide a basis for further stages of design.

The next step for this project is to carry out a similar optimisation procedure for the baseline shell model by varying the shell thicknesses, in order to compare the trade study results of a more conventional parametric model with that of the evolutionary structural optimisation approach. Comparisons will be drawn between the design time, the design recommendations and the design performance achieved by each trade study.

\section{Acknowledgments}

The authors acknowledge the funding provided by Rolls-Royce to do this work. Permission to publish the work is also acknowledged. 


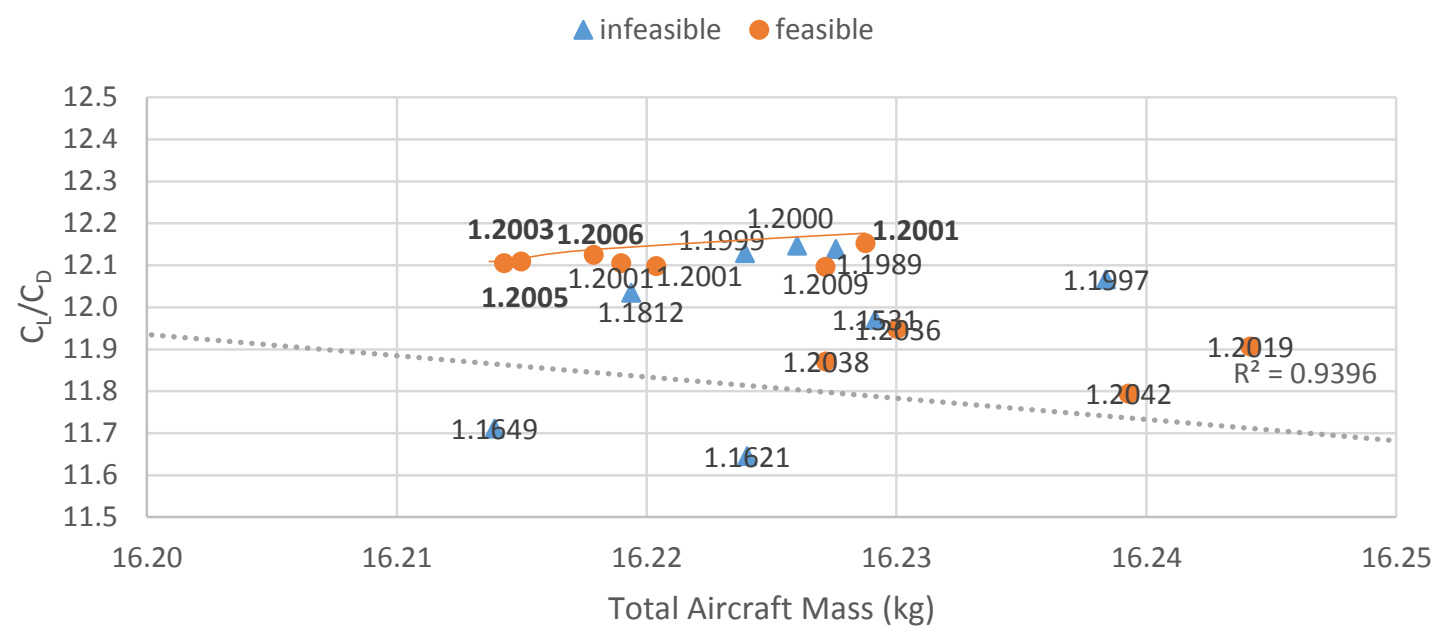

Figure 14 - Total Aircraft Mass vs. Aerodynamic Efficiency (Area of Interest)

\section{References}

${ }^{1}$ D. Gorissen, E. Quaranta, M. Ferraro, J. Scanlan, A. Keane, and K. Takeda, "Architecting a Decision Environment for Complex Design Evaluation," 53rd AIAA/ASME/ASCE/AHS/ASC Structures, Structural Dynamics and Materials Conference, American Institute of Aeronautics and Astronautics, 2012.

${ }^{2}$ A. J. Keane and P. B. Nair, Computational approaches for aerospace design: the pursuit of excellence, Wiley, 2005.

${ }^{3}$ E. Quaranta, J. van Schaik, M. Ferraro, D. Gorissen, J. Scanlan, and A. Keane, "Aerodynamic Optimization Integrated in Design of an Unmanned Aerial Vehicle," University of Southampton, 2012 (unpublished).

${ }^{4}$ J. Mariens, A. Elham, and M. van Tooren, "Influence of weight modelling on the outcome of wing design using multidisciplinary design optimisation techniques," Aeronautical Journal, Vol. 117, No. 1195, 2013, pp. 871-895.

${ }^{5}$ A. Sóbester and A. J. Keane, "Multidisciplinary design optimization of UAV airframes," 2nd AIAA Multidisciplinary Design Optimization Specialist Conf., 2006.

${ }^{6}$ M. D. Sensmeier and J. A. Samareh, "Automatic aircraft structural topology generation for multidisciplinary optimization and weight estimation," Proceedings of 46th AIAA/ASME/ASCE/AHS/ASC Structures, Structural Dynamics $\backslash \&$ Materials Conference, 2005.

${ }^{7}$ L. Krog, A. Tucker, M. Kemp, and R. Boyd, "Topology Optimisation of Aircraft Wing Box Ribs," 10th AIAA/ISSMO Multidisciplinary Analysis and Optimization Conference, 2004.

${ }^{8}$ M. Tomlin and J. Meyer, "Topology Optimisation of an Additive Layer Manufactured (ALM) Aerospace Part," The 7th Altair CAE Technology Conference, Altair Engineering, 2011.

${ }^{9}$ M. Muir, "Multidisciplinary Optimisation of a Business Jet Main Exit Door Hinge for Production by Additive Manufacturing," The 8th Altair Technology Conference, Altair Engineering, 2013.

${ }^{10}$ A. Remouchamps, M. Bruyneel, C. Fleury, and S. Grihon, "Application of a bi-level scheme including topology optimization to the design of an aircraft pylon," Structural and Multidisciplinary Optimization, Vol. 44, No. 6, 1 Dec. 2011 , pp. 739-750.

${ }^{11}$ D. M. Watts and R. J. M. Hague, "Exploiting the Design Freedom of Rapid Manufacture," Proc. 17th Solid Freeform Fabrication Symposium, 2006.

${ }^{12}$ X. Huang and Y. M. Xie, Evolutionary Topology Optimization of Continuum Structures: Methods and Applications, John Wiley \& Sons, 2010.

${ }^{13}$ X. Huang, Y. M. Xie, and M. C. Burry, "A new algorithm for bi-directional evolutionary structural optimization," Jsme International Journal Series C-Mechanical Systems Machine Elements and Manufacturing, Vol. 49, No. 4, Dec 2006, pp. 1091-1099.

${ }^{14}$ J. Deaton and R. Grandhi, "A survey of structural and multidisciplinary continuum topology optimization: post 2000," Structural and Multidisciplinary Optimization, 4 Jul. 2013, pp. 1-38.

${ }^{15} \mathrm{X}$. Huang and Y. M. Xie, "Convergent and mesh-independent solutions for the bi-directional evolutionary structural optimization method," Finite Elements in Analysis and Design, Vol. 43, No. 14, Oct 2007, pp. 1039-1049.

${ }^{16}$ ESDU, Full-Potential (FP) Method for Three-Dimensional Wings and Wing-Body Combinations - Inviscid Flow Part 1: Principles and Results, ESDU-02013, London, 2002.

${ }^{17}$ ESDU, Full-potential method for three-dimensional wings and wing-body combinations - inviscid flow. Part 4: Evaluation of trailing-vortex drag and wave components., ESDU-06016, London, 2006. 